\title{
Khon San - eine Fallstudie zu Naturpotenzial und Agrarkolonisation in Nordostthailand
}

\author{
Karl-Heinz Pfeffer, Tübingen, Sekson Yongvanit, Khon \\ Kaen
}

\section{Nordostthailand in der zweiten Hälfte des letzten Jahrhunderts}

Eingriffe in tropische Monsunwaldgebiete durch Holzeinschlag und eine ungelenkte, teils illegale Agrarkolonisation sind bekannte Ökosystemveränderungen in Südostasien (Uhlıg 1984). Für Isaan, die an Laos und Kambodscha angrenzende $200.000 \mathrm{~km}^{2}$ große Nordostprovinz Thailands, erfolgte seit dem Ende des Zweiten Weltkrieges ein rasanter Wandel (LÖFFLER \& MAAS 1992; Kunurat 1987). 1956 waren noch $61,5 \%$ des Khoratplateaus «Wald und Weideland» (PENDleton 1963), und bei einer Bevölkerung von 7,17 Millionen Einwohnern betrug die Fläche der Reisoasen nur $21.600 \mathrm{~km}^{2}$ und $592 \mathrm{~km}^{2}$ Feldfrüchteanbauland entlang der Hauptflüsse. Heute ist Isaan eine Agrarregion mit nur 12,43\% (1998) Waldbedeckung und einer Bevölkerung von rund einem Drittel der 60 Millionen (1998) Thailänder (YongVanit 2001). Dieser rasche Wandel hat an vielen Stellen $\mathrm{zu}$ ökologischen Schäden geführt, die bereits wieder zur Aufgabe und Umwandlung der Agrarflächen zwangen und Arbeitsmigration zur Folge hatten.

Im Rahmen eines deutsch-thailändischen Forschungsprojektes, finanziell unterstützt durch die Deutsche Forschungsgemeinschaft, die Gesellschaft für Technische Zusammenarbeit und den National Research Council des Kingdom of Thailand, wurden im Zeitraum 1994-1999 in Fallstudien Daten zu den zwei Bezirken Amphoe Khon San (Yongvanit 2001) und Amphoe Phu Pha Man (Schell 2001) am Westrand des Khoratplateus gewonnen. Archivarbeiten, insbesondere auch Auswertungen der in Thai verfassten amtlichen Berichte und Chroniken, Befragungen der Bewohner der Region und Kartierungen des Reliefs, der Böden, der Vegetation und des Anbaus sowie Laborarbeiten zur Bodenchemie lieferten die entsprechenden Grundlagen.

Die Zielsetzung war es, über die Geschichte der Agrarkolonisation, des Nutzungswandels und die ökologischen Auswirkungen sowie die heutigen sozioökonomischen Strukturen eine geoökologischsozioökonomische Bilanz als Grundlage für Handlungsstrategien zu erstellen.

\section{Amphoe Khon San}

Amphoe (Bezirk) Khon San (Abb. 1), $120 \mathrm{~km}$ westlich von Khon Kaen am Westrand des Khoratplateaus und dem Anstieg zu den Petchaboon Mountains, umfasst eine Fläche von $967 \mathrm{~km}^{2}$ mit 8 Tambons (Gemeinden) und 47 Ban (Dörfern) mit 10.460 Haushalten und 49196 Personen. Bis auf gesondert geschützte Bereiche ist die Region waldfreies Agrarland (Yongvanit 2001).

Noch in den fünfziger Jahren war der westliche Teil des Khoratplateaus bis auf kleine Rodungsinseln bewaldet. Im an den heutigen Ort Khon San angrenzenden westlichen Bergland gab es nur drei Siedlungen, von denen zwei im Rahmen von strategischen Maßnahmen während des Vietnamkrieges umgesiedelt wurden. Zwar ist die Situation der fünfziger Jahre statistisch nicht fassbar, aber die aus amtlichen Berichten und eigenen Erhebungen erstellte Besiedlungsgeschichte (Tab. 1) zeigt die enge Vernetzung des Raumes mit umgebenden Waldgebieten. Ausgangspunkt für die Entwicklung war die bereits 1800 an einem Flusslauf gegründete Siedlung Khon San, von der aus an durch Quellaustritte günstigen Stellen der Westrand des Khoratplateaus punktuell bis 1950 besiedelt wurde. Der Bevölkerungsdruck der sechziger Jahre - die Bevölkerung Thailands hatte sich von 1950 mit 17,4 Millionen bis 1970 mit 36,3 Millionen verdoppelt und der Bau strategischer Straßen ermöglichten eine weitgehend illegale Ausweitung der Besiedlung ohne Landbesitztitel an Straßensiedlungsleitlinien in das Gebirgs- und Waldland. Die Zuwanderer kamen in erster Linie aus Khon San und aus anderen Orten des Khoratplateaus. Die Nutzung des gerodeten Waldgebietes und der angrenzenden Waldflächen unterlag, von Selbstversorgung und Absatzmöglichkeiten bestimmt, einem raschen Wandel, der nach der Eröffnung von zwei Zuckerfabriken (1972 in Nam Phong und 1979 in Phu Khieo) und den Anbaufördermaßnahmen durch die Zuckerfabriken zur vollständigen Beseitigung der Waldflächen führte (Yongvanit 2001).

17 der in die Waldgebiete hineingewachsenen bzw. erst in den Waldgebieten entstandenen Dörfer des heutigen Amphoe Khon San wurden näher untersucht und jeweils zehn Haushalte interviewt. Aus den umfangreichen Datensätzen (Yongvanit 2001) sind für drei Dörfer die beigegebenen Tabellen zur Dokumentation der textlichen Darstellungen erstellt worden (Tab. 2). 


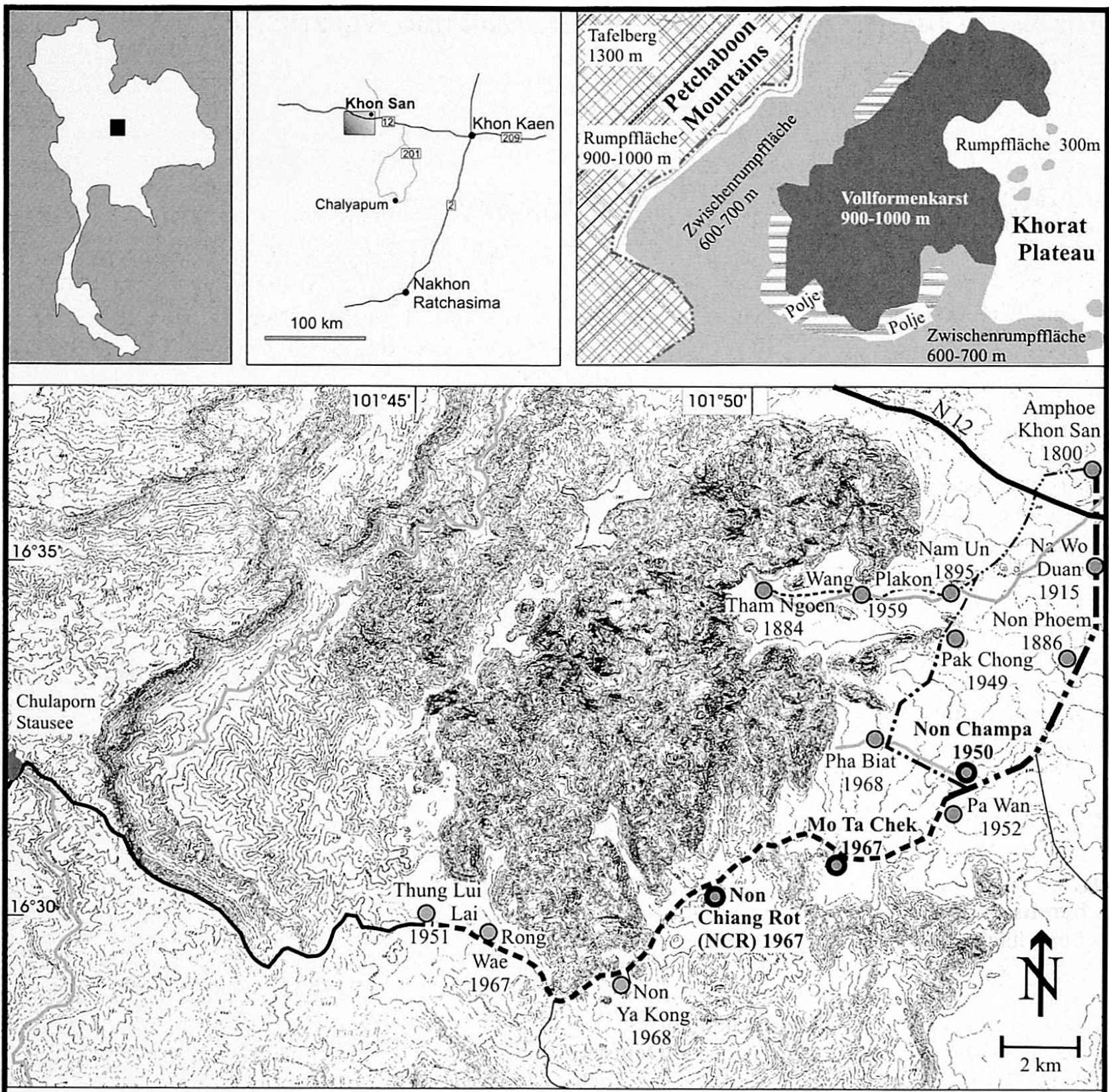

Fussweg seit 1895

Von 1895-1962 Fussweg

Neuere Verbindungsstrasse unklassifiziert
Seit 1962 Strasse bis Pak Chong, seit 1968 bis Pa Wan

Seit 1957 Lateritweg

von Pa Wan nach Thung Lui Lai seit 1974 asphaltiert

Highway N12 nach Petchaboon, 1979 erbaut seit 1974 asphaltiert

Seit 1968 Strasse

zum Chulaporn Staudamm, seit 1974 asphaltiert

Ort mit Gründungsjahr

- Beispielorte

Abb. 1: Amphoe Khon San: Lage, Topographie, Relief, Strassenbau, ausgewählte Ortsgründungen Amphoe Khon San: position, topography, relief, road construction, selected village foundations Amphoe Khon San: situation, topographie, relief, construction routière, choix de créations villageoises Quelle: Eigene Erhebungen; Kartengrundlage:Topographische Karte 1:50'000 des Königreiches Thailand; Kartographie: K.-H. Pfeffer, S. Yongvanit 


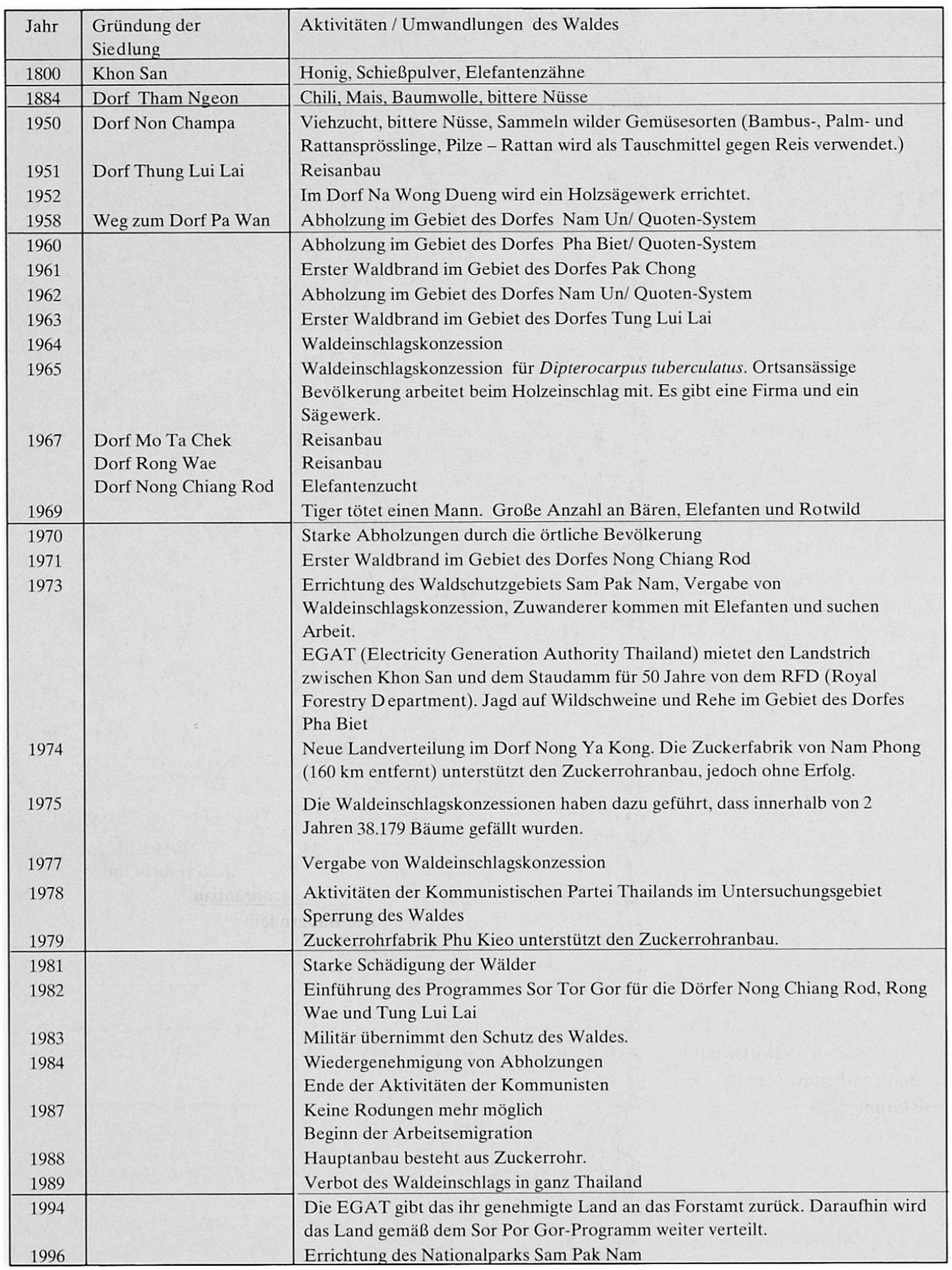

Tab. 1: Ausgewählte Daten zur Siedlungsgeschichte des Bezirks Khon San Selected data regarding housing development in the Khon San region

Choix de données statistiques relatives à l'histoire de la colonisation du district de Khon San

Quelle: Eigene Erhebungen und Daten aus amtlichen Berichten: Forstabteilung Chayaphom 1996; Khon San Agricultural Section 1995; Regionale Forstabteilung Nakhon Ratchasima 1973, 1974,1977-1980,1983-1985, 1989; Royal Forestry Department 1984, 1991, 1993, 1994, 1996, 1998. 


\begin{tabular}{|l|l|}
\hline \multicolumn{2}{|l|}{ Non Champa } \\
\hline 1950 & $\begin{array}{l}\text { 3 Familien aus Khon San, für Viehzucht } \\
\text { wurde Grasland genutzt. Die Bevölkerung } \\
\text { betreibt Jagd, sammelt wilde Gemüse- } \\
\text { sorten wie Bambus-, Palm- und Rattan- } \\
\text { sprösslinge sowie Pilze. Rattan wird als } \\
\text { Tauschmaterial gegen Reis verwendet. Es } \\
\text { wurde Land in Anspruch genommen, } \\
\text { hauptsächlich für Viehzucht. }\end{array}$ \\
\hline 1967 & $\begin{array}{l}\text { 4-5 Rai Wald wurden gerodet, um Felder } \\
\text { für den Anbau von Baumwolle und Chili } \\
\text { zu erhalten. }\end{array}$ \\
\hline 1970 & $\begin{array}{l}\text { 20 Rai Waldfläche wurde gerodet und } \\
\text { erschlossen für den Futtermaisanbau. }\end{array}$ \\
\hline 1975 & Die Zuwanderer kamen aus Khon San. \\
\hline 1977 & $\begin{array}{l}\text { Umwandlung einiger Felder in Reisfelder, } \\
\text { um die Eigenproduktion an Reis zu } \\
\text { erhöhen. }\end{array}$ \\
\hline 1984 & Arbeitsemigration \\
\hline 1996 & 144 Haushalte, 696 Einwohner \\
\hline
\end{tabular}

Landbesitz (Haushalte)

\begin{tabular}{|c|c|c|c|}
\hline $1-5 \mathrm{Rai}$ & $6-10 \mathrm{Rai}$ & $11-20 \mathrm{Rai}$ & $21-50 \mathrm{Rai}$ \\
\hline 50 & 24 & 40 & 30 \\
\hline
\end{tabular}

Landbesitzrecht (Haushalte)

\begin{tabular}{|c|c|}
\hline keine Dokumentation & kein Land \\
\hline $\mathrm{xxx}$ & 10 \\
\hline
\end{tabular}

Sor Por Gor ist ein Nutzungsrecht für das verteilte Grundstück. Die Besitzer haben ein Nutzungsrecht, aber kein Verkaufsrecht. Die Bank gibt keine Kredite für die Grundstücke. Das Nutzungsrecht ist vererbbar. Das Ministerium für Landwirtschaft und Zusammenarbeit ist verantwortlich für diese Grundstücke.

1 Rai $=40 \times 40 \mathrm{~m}$

\begin{tabular}{|l|l|}
\hline \multicolumn{2}{|l|}{ Mo Ta Chek } \\
\hline 1967 & $\begin{array}{l}\text { Gründung des Dorfes, Zuwanderer kamen } \\
\text { aus Khon San; hauptsächliche Nutzung: } \\
\text { Reisanbau zur Selbstversorgung, pro } \\
\text { Haushalt wurden 2-3 Rai kultiviert. }\end{array}$ \\
\hline 1969 & Fast jeder Haushalt pflanzt Futtermais. \\
\hline 1976 & 12 Familien Zuwanderer aus Khon San \\
\hline 1977 & $\begin{array}{l}\text { Zuwanderer aus Petchaboon, Chaiyaphom, } \\
\text { Nakhonratchasima und Nakhon Sawan }\end{array}$ \\
\hline 1986 & $\begin{array}{l}\text { Die Dorfbewohner verkaufen ihr Land, auf } \\
\text { dem sie nur illegal wohnen, für 5.000 } \\
\text { Baht/Rai an einen Zuckerunternehmer. Die } \\
\text { Bewohner, die kein Land zu verkaufen } \\
\text { hatten, suchten sich Arbeit als Land- } \\
\text { arbeiter oder wanderten in eine andere } \\
\text { Provinz oder nach Bangkok ab. }\end{array}$ \\
\hline 1996 & 87 Haushalte, 460 Einwohner \\
\hline
\end{tabular}

Landbesitzrecht

\begin{tabular}{|l|l|}
\hline Sor Por Gor & Kein Land \\
\hline $\mathrm{xxx}$ & 65 Haushalte \\
\hline
\end{tabular}

\begin{tabular}{|l|l|}
\hline \multicolumn{2}{|l|}{ NCR Tai (Nong Chiang Rod - Tai) } \\
\hline 1967 & $\begin{array}{l}\text { 3 Familien aus Khon San, Elefanten- } \\
\text { züchter, hauptsächlich Elefantenzucht }\end{array}$ \\
\hline 1974 & $\begin{array}{l}\text { Zuwanderer aus Khon San. Es waren } \\
\text { Elefantenzüchter, die mit einer Holzfabrik } \\
\text { zusammenarbeiteten. }\end{array}$ \\
\hline 1976 & $\begin{array}{l}\text { Die EGAT (Electricity Generation } \\
\text { Authority Thailand) gründete ein Dorf mit } \\
\text { 170 Einwohnern, dabei bekam jeder } \\
\text { Haushalt 15 Rai Land zugeteilt. }\end{array}$ \\
\hline 1979 & $\begin{array}{l}\text { Die Phu Khieo-Zuckerfabrik unterstützt } \\
\text { den Zuckerrohranbau. }\end{array}$ \\
\hline 1989 & $\begin{array}{l}\text { Die Bauern leihen sich Geld von der } \\
\text { Zuckerrohrfabrik, mit ihrem Land als } \\
\text { Pfand. }\end{array}$ \\
\hline 1991 & Arbeitsemigration \\
\hline 1997 & Zuckerrohranbau dominiert \\
\hline 1996 & 132 Haushalte, 644 Einwohner \\
\hline
\end{tabular}

Landbesitzrecht

\begin{tabular}{|l|l|}
\hline Keine Dokumentation & kein Land \\
\hline $\mathrm{xxx}$ & 20 \\
\hline
\end{tabular}

Tab. 2: Siedlungsgeschichte ausgewählter Dörfer

The housing development of selected villages

Histoire du peuplement d'un choix de villages

Quelle: Eigene Erhebungen und Daten aus amtlichen Berichten: Mo Ta Chek 1996; Non Champa 1996; Nong Chiang Rot-Tai 1996 


\section{Das Naturpotenzial des Agrarkolonisationsraumes}

Topographie und Relief: Zwei weitgespannte Rumpfflächen bestimmen das Relief. Die eine, in $300 \mathrm{~m}$ Höhe gelegene, bildet den Westrand des Khoratplateaus, während die andere in 900-1000 m die Hochfläche der Petchaboon Mountains bildet, lokal noch von Tafelbergen um $300 \mathrm{~m}$ überragt. Die Übergänge zwischen den beiden Rumpfflächen sind durch markante Stufen geprägt, an einigen Stellen ist noch ein Rumpfflächenzwischenniveau in 600-700 $\mathrm{m}$ zwischengeschaltet.

Die Rumpffläche der Petchaboon Berge wurde vom Khoratplateau aus buchtartig von Osten her aufgelöst, wobei Reste des Zwischenniveaus und auch Reste der Petchaboon Rumpffläche in kleineren isolierten Bergen und Bergzügen aus der Khoratrumpffläche herausragen. Tonsteine und dünnbankige Sandsteine bilden flache Kuppen und Rücken, während Kalke markante Kalkkuppen und Türme über der Khoratrumpffläche entstehen liessen.

Die Teile der Rumpffäche, die über Kalke hinwegzieht, sind verkarstet und in ein schwer zugängliches Vollformenkarstgebiet umgewandelt. Am Rande des Vollformenkarstes befinden sich kleinere Poljen, deren ebener Boden günstige Siedlungsflächen darstellt.

Die Khoratfläche ist eine weite flachwellige Rumpffläche, in die die großen Abflusssysteme aus den Petchaboon Mountains nur sehr wenig eingetieft sind.

Am westlichen Rande zu den höheren Reliefteilen der Zwischenniveau- und der Petchaboonrumpffläche treten an den Stufenhängen ganzjährig schüttende Karstquellen aus.

Die höhere Rumpffläche ist Teil des Nam Nao Nationalparkes, die Staudammregion ist Schutzgebiet der Elektrizitätswerke und der Vollformenkarst als Sam Phak Nam Nationalpark ausgewiesen. Diese Gebiete sind geschützte Waldgebiete und erlauben Vergleiche zu den Agrarregionen.

Böden: Die geomorphologische Geschichte bestimmt das Raummuster der Böden. Die Böden auf den Rumpfflächen waren lateritische Verwitterungsresiduen mit Dominanz des Kaolinittonminerals. Auf der Khoratrumpffläche waren die Vorkommen flächendeckend, im Bereich der Petchaboonfläche gab es nur bei grösseren meist über Sandsteinen erhaltenen - Flächenresten eine grössere Verbreitung dieses Bodentyps.

Nur im A-Horizont besteht eine hohe Kationenaustauschkapazität und eine gute Nährstoffversorgung. Bereits im gebleichten Eluvialhorizont nimmt entweder die Kationenaustauschkapazität stark ab, oder die
Nährstoffversorgung geht stark zurück. Die tieferen Horizonte bieten für Pflanzen sehr ungünstige Parameter.

Die Böden an den Talhängen im Bereich der Petchaboon Berge sind Acrisole. Es gibt im B-Horizont noch günstige Kationenaustauschkapazitätswerte, aber in den Nährstoffen liegen bereits deutliche Verarmungen und ein stark saurer $\mathrm{pH}$ vor. Diese Böden sind besser geeignet für eine landwirtschaftliche Nutzung, aber auch hier konzentrieren sich die für das Pflanzenwachstum günstigen Parameter auf den obersten Bodenpartien.

Auf den Kalkkuppen fehlt nahezu jegliche Bodenbedeckung, nur an den weniger steilen Kuppenhängen, an den Flanken und Hangschleppen zu den Poljen und zur Khoratrumpffläche wurden Cambisole mit sehr guten Werten für die Nährstoffversorgung und die Kationenaustauschkapazität kartiert.

Bei allen untersuchten Böden belegen sehr weite $\mathrm{C} / \mathrm{N}$ Verhältnisse die eingeschränkte N-Verfügbarkeit und mobiles Eisen verursacht Mangel an Phosphor.

Alle Böden zeigen auf den entwaldeten Flächen eine hohe Erosionsanfälligkeit.

Klima: Die Auswertungen der Klimaaufzeichnungen (Yongvanit 2001) ergaben jährliche Durchschnittstemperaturen auf der Khoratrumpffäche zwischen $25^{\circ}$ bis $27^{\circ}$. Über das Jahr hin ergeben sich die typischen Schwankungen der Stationen im Monsunklima. Mit dem Einsetzen der trockenen Jahreszeit sinken die Monatsdurchschnittstemperaturen von November bis Februar auf $21^{\circ}$ bis $23^{\circ}$, um in der Vormonsunzeit im April und Mai dann mit Monatsdurchschnittstemperaturen von $28^{\circ}$ bis $30^{\circ}$ die höchsten Werte zu erreichen. Die Sommermonate liegen bei $27^{\circ}$ bis $28^{\circ}$ Monatsdurchschnittstemperatur.

Für die Petchaboon Mountains lässt sich aus der höheren Lage mittels der Formel nach LaUtenSACH \& BöGEL in BLÜTHGEN (1966: 118) kalkulieren, dass auf den Ebenheiten in $900 \mathrm{~m}$ Höhe mit Durchschnittstemperaturen zwischen $17^{\circ}$ und $19^{\circ}$ in der Trockenzeit und $24^{\circ}$ bis $25^{\circ}$ in der feuchten Zeit zu rechnen ist.

Das Vorland der Petchaboon Mountains weist Niederschlagshöhen von 800 bis $1000 \mathrm{~mm} / \mathrm{Jahr}$ auf, während die Gebirgsstationen 1100 bis $1700 \mathrm{~mm} / \mathrm{Jahr}$ aufweisen.

Berechnungen zur Humidität zeigen, dass bis zum Einsetzen der Monsunregen Wassermangel herrscht, während über die gesamte Regenzeit humide bis perhumide Bedingungen bestehen. 
Die Trockenperiode auf der Khoratrumpffläche beträgt fünf Monate, während sich die Trockenperiode in den feuchteren Gebirgsstationen auf drei Monate reduziert.

Die feuchte Jahreszeit bietet in allen Regionen des Untersuchungsgebietes einen hohen Überschuss an Wasser. Dies führt zu starken Abflüssen in den Regenmonaten, und die Abflussrinnen der Khoratrumpffläche und die Alluvialebenen der vom höheren westlichen Relief kommenden Flüsse stehen unter Wasser. Die Gebirgsbäche führen nahezu ganzjährig dem Vorland Wasser zu und Karstquellen am westlichen Rand der Khoratrumpffläche schütten ganzjährig ergiebig.

Vegetation: Die Vegetation zeichnet den monsunalen Gang der Niederschläge nach und ist an die unterschiedlich langen humiden Bedingungen angepasst.

Die Naturwälder sind laubwerfende Dipterocarpaceenwälder im Vorland und im Anstieg der Petchaboon Mountains, während die Karstgebiete und die Petchaboon Mountains ab ca. $700 \mathrm{~m}$ Höhe halbimmergrüne Dipterocarpaceenwälder aufweisen. Sie sind auf der Höhe der Petchaboon Mountains mit Kiefernwald durchsetzt (WeRner 1993; YongVANIT 2001).

Auf der Khoratrumpffläche sind nur innerhalb der Klostergebiete Waldbestände vorhanden. Die Pflanzen und Baumarten deuten darauf hin, dass die naturnahe Vegetation ein laubwerfender Dipterocarpaceenwald war.

Fazit für eine agrare Nutzung: Die Rumpfflächen sind schwach reliefiert und für den Einsatz von Maschinen gut geeignet. Die Rumpfstufe und die in den Tonstein- und dünnbankigen Sandsteinen stark zerschnittene Petchaboonrumpffläche sowie die Talschichtstufe weisen steile Hänge auf, die zwar landwirtschaftlich genutzt werden könnten, aber der Einsatz von Maschinen ist schwierig, zum Teil auch unmöglich. Die Vollformkarstgebiete sind sehr schwer zugänglich und nur bedingt nutzbar. Einzelkuppen sind für den Anbau ungeeignet. Die Böden der Rumpfflächen sind für eine agrarische Nutzung nicht günstig, sowohl vom Nährstoffhaushalt her als auch von der Erosionsanfälligkeit der Oberböden. Der darunter vorhandene Plinthit verhärtet an der Oberfläche nach der Erosion des E-Horizontes zu einer Lateritkruste. Die Böden der Hanglagen sind vom Nährstoffhaushalt günstiger, bei anstehendem Kalkgestein sogar als gut für eine landwitschaftliche Nutzung einzustufen. Die Korngrößen der Böden sprechen für eine hohe Erosionsanfälligkeit, die geringe Bodenmächtigkeit erlaubt keine großen Erosionsraten, da sonst sehr rasch das anstehende Gestein die Oberfläche bildet. Die Wasserbilanz lässt die Gebirgsregionen für den Anbau günstiger erscheinen, das Tiefland ist aber durch die feuchte Jahreszeit mit dem Wasserüberangebot und der Zufuhr von Wasser aus den Hochgebieten und Karstquellen besser für den Anbau geeignet. Gefährdend für die agrarisch genutzten Böden ist, dass nach der Trokkenzeit auf die nicht von der Vegetation geschützten Böden die mit Starkregen einsetzenden Monsunregen eine große Erosionskraft haben.

\section{Sozioökonomische Analyse der heutigen Strukturen}

Heute ist das Gebiet des Petchaboon Gebirges im Bereich des Nam Nao-Nationalparks und der angrenzenden National Forest-Gebiete ein grossräumig geschütztes Waldgebiet. Um die Siedlungen herum ist der Wald vollständig verschwunden und in den Ebenen dominiert in den Tiefenzonen Reis, während Zuckerrohr großflächig die jahreszeitlich nicht inundierten Flächen einnimmt (Abb. 2). In Hanglagen der Petchaboon Berge wird auch Mais großflächig angebaut. Kleinere Areale mit Obst und Maulbeeren (Seidenraupenzucht) sind in die Flur eingestreut. Die Obstbaumkulturen sind jüngere Anpflanzungen. Eukalyptusaufforstungen - in der Statistik zum Wald gerechnet - sind im Amphoe Khon San im Gebiet des National Forest zum Chulaporn Dam hin in aufgelassenen Ackerflächen zu beobachten. In allen Siedlungen gibt es Hausgärten, die bei Siedlungen an Karstquellen hohe Einahmen ermöglichen.

Die Analyse der heutigen Strukturen und der Landnutzung (Tab. 3 und 4) zeigen einen Agrarraum mit einer flächendeckenden Nutzung aller Ressourcen.

Die Geländebefunde zeigen, dass ökologische Fragestellungen unberücksichtigt sind und dass selbst auf ungünstigsten Flächen angebaut wird. Auch die Waldgebiete werden, ohne Erlaubnis, zur Erzielung von Einkünften genutzt. Es gibt nur in wenigen Fällen verbrieften Landbesitz. Kapital für einen Wechsel der Anbaumethoden oder der Produkte ist nicht vorhanden. Die fehlenden Besitztitel erlauben keine Kapitalaufnahmen bei Banken, vielmehr wird privat Geld geliehen. Über eine Arbeitsmigration nach Bangkok wird versucht, zusätzliche Einnahmen zu gewinnen.

Die Zahlen geben Einblick in die Strom- und Wasserversorgung sowie in die Infrastruktur bezüglich der Schulen. Sie belegen, dass die Einkünfte zum täglichen Leben zwar reichen, dass aber die von außerhalb der Region kommenden Gelder unbedingt erforderlich sind. Doch auch dieses Kapital reicht nicht aus, die im Rahmen der Besiedlung für den Hausbau, Geräte oder Saatgut und Pflanzen entstandenen Schulden abzuzahlen.

Insgesamt zeigt sich, dass trotz der weitgehend semile- 


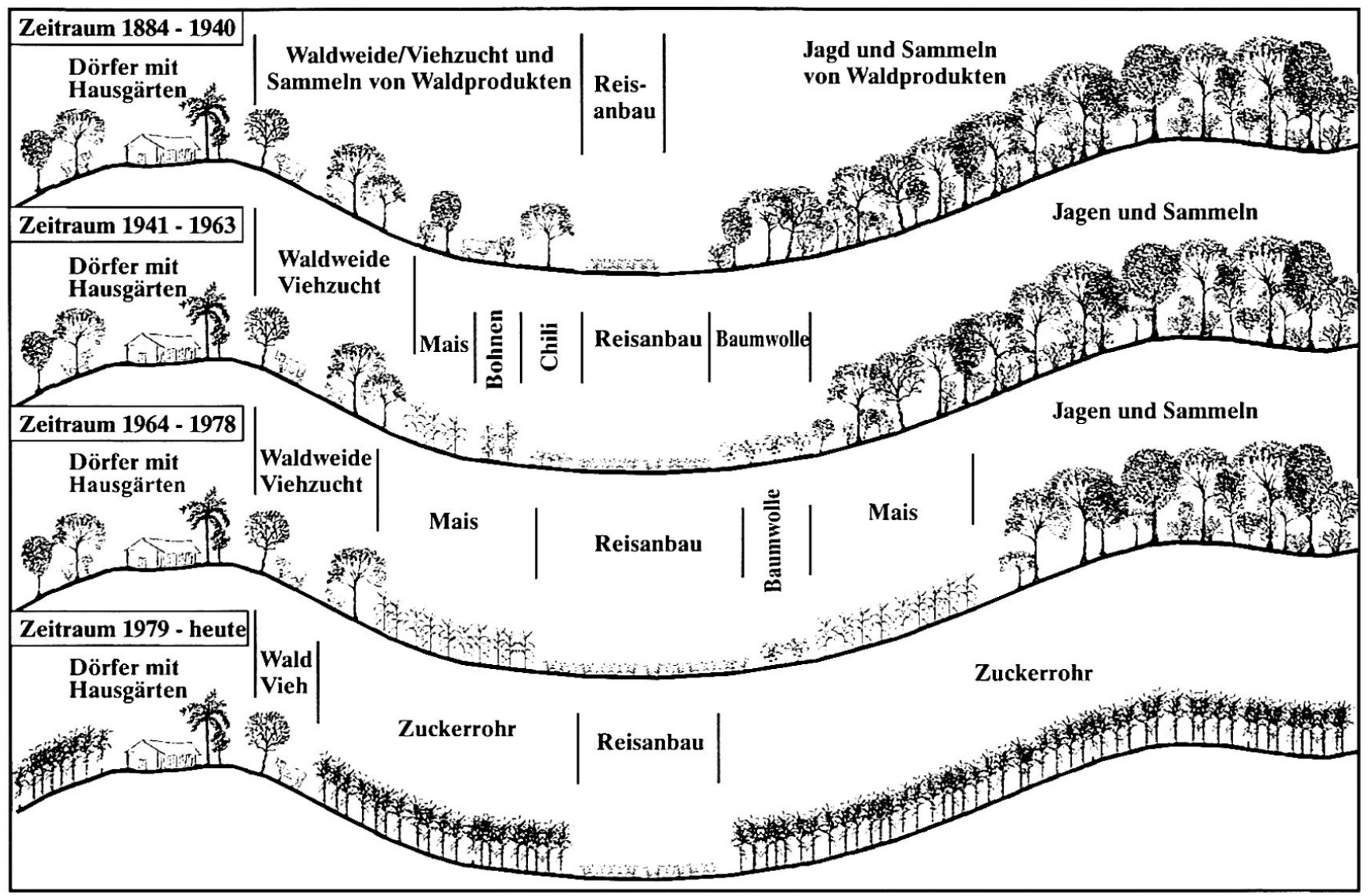

Abb. 2: Wandel in der Agrarlandschaft

Agricultural change

Mutation du paysage agraire

Graphik: K.-H. PfEFfER

galen-illegalen Besiedlung die Grundversorgung gesichert ist und die Bewohner sich ernähren können. Die Erwachsenengeneration hat einen sehr niedrigen Bildungsstand, ist weitgehend ohne Landbesitztitel und verschuldet. Insgesamt zeigt sich eine Situation, die eine Änderung vorhandener Strukturen nur durch Maßnahmen von «außen» erlaubt.

\section{Prozesse der Ökosystemschädigung}

Die langen Aufenthalte im Untersuchungsgebiet zu allen «monsunalen» Jahreszeiten zeigten, dass sowohl die manuellen als auch die maschinellen Anbautechniken und der Zwang, auch ungünstige Standorte wie steile Hanglagen, auf Kalk mit nur dünner Bodenbedeckung zu nutzen, das Naturpotenzial nachhaltig geschädigt haben und sich diese Dynamik fortsetzt.

Wesentlich ist, dass der Einsatz von Feuer zur Vorbereitung der Feldbestellung bei den herrschenden Kli- mabedingungen und der Bodenstruktur gravierende, rasch ablaufende Prozesse in den Hanglagen auslöst.

Nach dem Abernten der Felder ist im Spätherbst zu Beginn der Trockenperiode immer noch genügend Feuchtigkeit im Boden vorhanden, dass vorhandenes Samenpotenzial des Imperata cylindrica-Grases keimen und aufwachsen kann. Bis Februar/März überzieht ein bis zu $2 \mathrm{~m}$ hoher Graswuchs die Feldflur. Um eine Neubestellung zu ermöglichen, wird das Gras abgebrannt und vorhandener Boden umgebrochen und eingesät. Ob kleine Motorfräsen oder durch Traktoren gezogene große Pfluggeräte (im Lohnauftrag von nicht im Gebiet ansässigen Kreditgebern) eingesetzt werden, mit Beginn der Monsunregenzeit liegt die Bodenkrume ungeschützt da und jedes einzelne Niederschlagsereignis hinterlässt deutliche Erosionsrinnen im Hang. Bei Tonsteinen und dünnbankigen Sandsteinen entstehen innerhalb von wenigen Niederschlagsereignissen bis zu einem Meter tiefe Rinnen, während in Kalkgebieten das feste Kalkgestein freigelegt wird. 


\begin{tabular}{|c|c|c|c|c|}
\hline & Dorfname: & Mo Ta Chek & Non Champa & NCR (TAI) \\
\hline 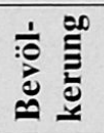 & $\begin{array}{l}\text { Männlich } \\
\text { Weiblich } \\
\text { Summe }\end{array}$ & $\begin{array}{l}187 \\
273 \\
460\end{array}$ & $\begin{array}{l}355 \\
341 \\
696\end{array}$ & $\begin{array}{l}325 \\
319 \\
644\end{array}$ \\
\hline 京蓄 & $\begin{array}{l}\text { 4 Jahre } \\
9 \text { Jahre } \\
\text { 12 Jahre } \\
\text { > 12 Jahre }\end{array}$ & $\begin{array}{c}\text { k. Angabe } \\
3 \\
4 \\
1\end{array}$ & $\begin{array}{r}300 \\
50 \\
2 \\
1\end{array}$ & $\begin{array}{r}450 \\
30 \\
0 \\
0\end{array}$ \\
\hline 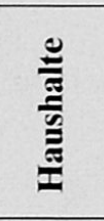 & $\begin{array}{l}\text { Anzahl } \\
\text { Stromversorgung } \\
\text { Trinkwasser } \\
\text { Wasserleitung } \\
\text { ohne Landbesitz } \\
\end{array}$ & $\begin{array}{r}87 \\
87 \\
75 \\
0 \\
65\end{array}$ & $\begin{array}{r}144 \\
140 \\
144 \\
0 \\
10\end{array}$ & $\begin{array}{r}132 \\
132 \\
102 \\
102 \\
20 \\
\end{array}$ \\
\hline 竞高 & $\begin{array}{l}\text { Anzahl betroffener } \\
\text { Haushalte } \\
\text { Männlich } \\
\text { Weiblich } \\
\text { Ziel }\end{array}$ & $\begin{array}{r}6 \\
10 \\
7\end{array}$ & $\begin{array}{r}5 \\
3 \\
2 \\
\text { Bangkok } \\
\end{array}$ & $\begin{array}{l}22 \\
23 \\
18\end{array}$ \\
\hline 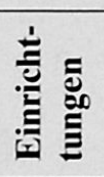 & $\begin{array}{l}\text { Kindergarten } \\
\text { Grundschule } \\
\text { Gesundheits- } \\
\text { station }\end{array}$ & $\begin{array}{l}1 \\
1 \\
1\end{array}$ & $\begin{array}{l}0 \\
1 \\
1\end{array}$ & $\begin{array}{l}1 \\
1 \\
1\end{array}$ \\
\hline 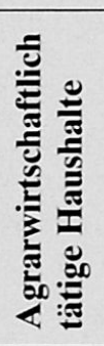 & $\begin{array}{l}\text { Reisbau } \\
\text { Sojabohnen } \\
\text { Rote Bohnen } \\
\text { Süßmais } \\
\text { Zuckerrohr } \\
\text { Landarbeiter } \\
\text { Haushalte } \\
\text { insgesamt }\end{array}$ & $\begin{array}{r}0 \\
0 \\
0 \\
0 \\
0 \\
87 \\
\\
0\end{array}$ & $\begin{array}{c}90 \\
54 \\
\text { k. Information } \\
10 \\
5 \\
0 \\
120\end{array}$ & $\begin{array}{r}5 \\
0 \\
100 \\
125 \\
0 \\
0 \\
\\
125\end{array}$ \\
\hline$\frac{y^{\frac{2}{2}}}{\frac{2}{2}}$ & $\begin{array}{l}\text { HH/ Anzahl Kühe } \\
\text { HH/ Anzahl Büffel } \\
\text { HH/Anzahl } \\
\text { Schweine } \\
\text { HH/ Anzahl } \\
\text { Geflügel }\end{array}$ & $\begin{array}{l}0 / 0 \\
0 / 0 \\
0 / 0 \\
0 / 0 \\
\end{array}$ & $\begin{array}{r}0 / 0 \\
0 / 0 \\
0 / 20 \\
\\
20 / 288 \\
\end{array}$ & $\begin{array}{c}7 / 40 \\
2 / \mathrm{k} \text {. Inform. } \\
5 / \mathrm{k} \text {. Inform. } \\
51 / \mathrm{k} \text {. Inform. }\end{array}$ \\
\hline
\end{tabular}

Tab. 3: Sozial- und Agrarstruktur

Social and agricultural structure

Structures sociales et agraires

Quelle: Eigene Erhebungen und Daten aus amtlichen Berichten: Mo Ta Chek 1996; Non Champa 1996; Nong Chiang Rot-Tai 1996

Als Folge davon ist an vielen Stellen zu beobachten, dass die Hänge frei von Bodenbedeckung sind und dass in den Tiefenlagen mehrere Meter mächtiges Kolluvium angeschwemmt ist.

Der Anbau an den Hängen erfolgt bei Tonsteinen und dünnbankigen Sandsteinen vielfach im anstehenden Gestein und im Falle von Kalk zwischen freigelegten Kluftkarren.
Auch in den geschützten Nationalpark- und National Forest-Bereichen wird Feuer gelegt. Dies geschieht einerseits, um sich vor Schlangen zu schützen, andererseits, um eine leichtere illegale Jagd auf Kleintiere zu ermöglichen und um die Sukzession zu beeinflussen. Auf den so entstandenen Brandflächen dominiert Bambus, der, ohne Erlaubnis entnommen, eine wichtige Einnahmequelle darstellt. Kalkhügel, Teile des Kegelkarst- 


\begin{tabular}{|l|c|c|c|}
\hline \multicolumn{1}{|c|}{ Dorfname: } & Mo Ta Chek & Non Champa & NCR (TAI) \\
\hline Agraranbau & 3.000 & 14.700 & 43.000 \\
\hline Landarbeit & 272 & 1.600 & 3.300 \\
\hline $\begin{array}{l}\text { Arbeit in der } \\
\text { Umgebung }\end{array}$ & 0 & 500 & 2.200 \\
\hline $\begin{array}{l}\text { Arbeit in } \\
\text { Bangkok }\end{array}$ & 0 & 270 & 10.000 \\
\hline $\begin{array}{l}\text { Sonstige } \\
\text { Arbeiten }\end{array}$ & 0 & 300 & 0 \\
\hline $\begin{array}{l}\text { Sonstige } \\
\text { Geldquellen }\end{array}$ & 890 & 5.000 & 3.400 \\
\hline $\begin{array}{l}\text { Waldnutzung } \\
\text { Hausgarten }\end{array}$ & $24.610^{2}$ & $41.500^{3)}$ & 5.850 \\
\hline $\begin{array}{l}\text { Summe des } \\
\text { Einkommens }\end{array}$ & 2.755 & 63.870 & 2.700 \\
\hline $\begin{array}{l}\text { Durchschnitt } \\
\text { der Investitio- } \\
\text { nen }\end{array}$ & 31.527 & 7.300 & 70.450 \\
\hline $\begin{array}{l}\text { Durchschnitt } \\
\text { des Saldos }\end{array}$ & 3.140 & 2.100 & 36.800 \\
\hline $\begin{array}{l}\text { Durchschnitt } \\
\text { der Schulden }\end{array}$ & 5.387 & 56.570 & 33.650 \\
\hline $\begin{array}{l}\text { NCR (TAI) } \\
\text { Nat }\end{array}$ & 500 & 00 \\
\hline
\end{tabular}

1) NCR (TAI) - hat bei hohen Investitionen hohe Einnahmen aus dem Zuckerrohranbau.

2) Mo Ta Chek - Einkünfte ohne Investitionen aus dem Wald durch Sammeln von Bambusholz und Bambussprösslingen

3) Non Champa - Einkünfte aus den von einer Karstquelle ganzjährig bewässerten Hausgärten

Bezirk Khon San (Haushalt-Grösse 5 )

Jahreseinkommen 32.965 Ausgaben 11.424

\begin{tabular}{|l|c|c|c|}
\hline $\begin{array}{l}\text { Vergleich: } \\
\text { Statistisches } \\
\text { Jahrbuch 1996 }\end{array}$ & Thailand & BKK & $\begin{array}{l}\text { Nordost- } \\
\text { region }\end{array}$ \\
\hline Einkommen & 99.912 & 198.516 & 68.220 \\
\hline Ausgaben & 96.408 & 167.940 & 73.608 \\
\hline HH-Grösse & 3.8 & 3.3 & 4.1 \\
\hline
\end{tabular}

Tab. 4: Einkünfte pro Haushalt im Jahr in Baht

Household income per year in Baht

Revenus/ménage et par an à Baht

Quelle: Eigene Erhebungen und Daten aus amtlichen Berichten: Mo Ta Chek 1996; Non Champa 1996; Nong Chiang Rot-Tai 1996

gebietes und straßennahe Waldstücke sind von Bambussekundärvegetation bedeckt.

Auf der Khoratrumpffläche führen selbst bei dem flachen Relief Spülrinnen zu den muldenförmigen, meist mit Reisanbau genutzten Tiefenlinien. Der Oberboden der Laterite wird abgetragen und der Plinthit ist an der Oberfläche verhärtet und bildet einen Eisenpanzer. An diesen Stellen sind nicht mehr genutzte Felder zu kartieren.

Die Befragungen von ausgewählten Standortnutzern ergab, dass nach ersten Agrarerfolgen die Erträge rasch zurückgingen und dass die Veränderungen der 
Standortverhältnisse zur Aufgabe von Anbauflächen führten.

\section{Die Folgen der Eingriffe des Menschen für das Ökosystem}

Bis auf die National Forest-Areale am westlichen Rand der Ebene ist die Khoratrumpffläche waldfrei. Primäre Wald- und Bodenstandorte sind nur noch in Tempelbezirken zu finden.

In den Reisanbaugebieten - in den Tiefenlinien der Abflussrinnen und in den unteren Hangbereichen wurde das Gelände terrassiert, wobei die feinsandigschluffigen Oberböden zur Hälfte ihrer Mächtigkeit zum Bau von die Felder umgebenden Dämmchen benutzt wurden. Die verbliebenen Teile des Oberbodens sowie die oberen Abschnitte der B-Horizonte weisen deutliche Kennzeichen einer Pseudovergleyung als Ergebnis der Nassreiskulturen auf. In den Tiefenlinien wurden auch Wasserbecken angelegt.

Die Rodung und Nutzung der flachen Rücken zwischen den Abflussrinnen, insbesondere der in jüngster Zeit durch Maschineneinsatz großflächig betriebene Zuckerrohranbau, haben zu Bodenerosion geführt, die Kolluvien in die Abflussrinnen und in die Hochwasserbecken der Flüsse eingeschwemmt hat. Auf den flachen Rücken zwischen den Abflussrinnen wurde der feinsandig-schluffige Oberboden erodiert und der Plinthithorizont an die Oberfläche gebracht. Dieser ist an der Oberfläche verhärtet und die landwirtschaftliche Nutzung wurde auf den flachen Rücken meist aufgegeben. Nur an wenigen Stellen konnte beobachtet werden, dass auf der zu Lateritkrusten verhärteten Oberfläche angebaut wurde. Weit verbreitet sind Eukalyptusmonokulturen, die von den Termiten gemieden werden und bereits nach fünf bis acht Jahren schneidfähig sind und in der Papier- und Zellstoffindustrie gute Einnahmen bringen. Diese Monokulturen werden als «Aufforstungen» bezeichnet. Nachteilig ist, dass das ätherische Öle enthaltende Laub bei der Zersetzung die Böden schädigt und künftige anderweitige Nutzungen mittelfristig ausschließt. Es gibt Proteste gegen «die den Boden vergiftenden» Aufforstungen durch NGO-Gruppen.

Die Hanglagen und Reliefteile der Tonsteine und dünnbankigen Sandsteine mit den Acrisolen werden großfächig als Agrarland genutzt und sind einer extremen Bodenerosion unterworfen. An vielen Stellen fehlen die Böden bis zum Anstehenden und Cv-Horizonte der Tonsteine und dünnbankigen Sandsteine werden durch Gullyerosion angegriffen. Spülrinnen und metertiefe Rinnen entstehen.
Kleinbauern sind nicht in der Lage, die Rinnen zu beseitigen, der Anbau von Gemüse und Mais erfolgt zwischen den Rinnensystemen. Beim Einsatz von Lohnpflügen wird mit unterschiedlichem Erfolg versucht, die Rinnen zuzupflügen. Dies geschieht im April, aber nach dem ersten Monsunregen im Mai entstehen bereits wieder neue Rinnen.

Die Karstgebiete haben nur eine kulturelle Bedeutung. Die großen Höhlenportale in den Kuppenhängen und am Kuppenfuß sind Meditationsplätze und kleine Tempelbezirke. Die äußerst schwer zugänglichen Regionen mit Einzelbäumen und dem hohen Anteil an Bambussekundärvegetation entziehen sich sonst einer agraren Nutzung, aber es gibt eine illegale Jagd. Um für die Jagd eine bessere Sicht zu haben und auch um Schlangen zu vertreiben, werden die Karstgebiete regelmäßig angezündet. Bestandsaufnahmen zeigten, dass an Positionen, die mehrere Jahre nicht angezündet wurden, eine Baumverjüngung möglich ist. Auf den häufig abgebrannten Gebieten fehlt eine Baumverjüngung, und die dominanten Bambusgewächse entwickeln einen dichten Wurzelfilz, so dass jedes weitere Aufkommen anderer Baumarten verhindert wird.

Die Poljeflächen sind uneingeschränkt Agrarflächen. Die tieferen Teile sind Reisanbauflächen, die trokkeneren Standorte werden für den Anbau von Gemüse und Obst genutzt. Neue Baumkulturen sowie Maulbeerbäume mit einer Seidenraupenzucht sind vereinzelt zu beobachten. Die die Poljen umgebenden Hänge wurden im Bereich der Tonsteine und dünnbankigen Sandsteine und der mit umgelagerten Tonsteinen bedeckten Kalkkuppenhangschleppen in die agrare Nutzung einbezogen. Dort sind die gleichen Erosionserscheinungen zu beobachten wie in den anderen Tonstein- und dünnbankigen Sandsteingebieten. Bei dünner Tonsteinbedeckung der Kalkhänge tritt der Kalk an die Oberfläche und die Agrarfläche bringt so wenig an Erträgen, dass nur noch vereinzelt eine Nutzung erfolgt.

Einige Ackerflächen und zugehörige squatter-Häuser sind seit 10 bis 15 Jahren nicht mehr genutzt. Die aufgelassenen Flächen bedeckt Unkraut, junge Bäume aus angrenzenden Waldrestarealen von den steileren nicht genutzten Kuppen sind stellenweise zu beobachten. Häufiger ist aber, dass auf den Feldflächen angelegte Feuer auf die mit Gras und Unkräutern bestandenen Hangschleppen übergreifen und damit jegliche Baumsukzessionen verhindern.

Die zwangsweise zum National Forest erklärten Tonstein- und dünnbankigen Sandsteingebiete sind mit Monokulturen aufgeforstet. Auf den aufgeforsteten Flächen dominieren die in der Nordostregion nicht 
beheimateten Eukalypten sowie Teak. Monokulturen, deren Auswirkungen auf das Ökosystem bei Teak nicht ersichtlich sind. Es konnten aber große Schädigungen durch Insektenbefall beobachtet werden. Die Eukalypten zeigen Veränderungen der Bodenparameter durch die ätherischen Zersetzungsprodukte der Blätter, im Umkreis der Pflanzung wachsen keine weiteren Pflanzen. Für die Auswirkungen auf den Wasserhaushalt im Boden und auf anorganische Bodenparameter sind die Zeitreihen der «Aufforstungen» aber noch nicht lange genug, um diese Veränderungen zu dokumentieren.

Bei den Aufforstungen mussten mangels der erodierten Böden die Pflanzen direkt in die Cv-Horizonte gesetzt werden. Die Pflanzen wachsen im Sinne einer möglichen Holznutzung rasch, aber die Bestände sind für Eukalyptus doch wenig ertragreich, da Bodenerosion, oberflächlicher Abfluss und Nährstoffaustrag ungünstige Standortfaktoren darstellen. Die Aufforstungen in den zum National Forest erklärten Gebiete mit der zwangsweisen Verlegung von Siedlungen und der Vertreibung einzelner squatter finden keine Akzeptanz, es wird häufig Feuer gelegt, und an vielen Stellen sind die Aufforstungen stark geschädigt. Auch werden die Felder der ehemaligen zwangsweise aus Waldgebieten umgesiedelten Dörfer illegal in den jetzigen Forstgebieten noch genutzt. Durch Feuer wird die illegale Anbaufläche vorbereitet. Dadurch kann keine Sukzession von den angrenzenden Waldbeständen aufkommen.

Die unter Schutz gestellten Waldgebiete auf der Petchaboon-Rumpffläche und den zugehörigen Hangarealen sind durch Zufahrtsschranken und bewaffnete ranger im Rahmen des Möglichen geschützt. Die Waldgebiete zeigten - bis auf die durch Holzlizenzen entfernten Baumriesen - ein recht intaktes Ökosystem. Es wird jedoch im Nationalpark und im National Forest immer wieder Feuer gelegt, um gute Jagdbedingungen für das Wildern zu schaffen. Dies führt besonders in den lichten Waldarealen mit hohen Kiefernanteilen zu Schädigungen der Bäume. Tropische Zyklone, die sich bis in die Petchaboon-Berge auswirken, brechen große Bestände der hohen, feuer- und pilzgeschädigten Bäume ab. Diese stehen als angekohlte, pilzüberzogene Strünke in den Kiefernwaldarealen.

\section{Synthese der physisch-geographischen und sozioökonomischen Betrachtungsebenen}

Die Petchaboon-Berge und ihr östliches Vorland sind ursprünglich ein von der Monsunzirkulation bestimmtes Waldland, das in weiten Teilen der Rumpfflächengebiete, der Tonstein- und dünnbankigen Sandsteinegebiete und in den Poljen illegal oder semilegal einer agraren Nutzung zugänglich gemacht wurde.
Die heute in dem Bezirk lebende Bevölkerung mit geringem Bildungsstand, überdurchschnittlich großen Haushalten und geringem Besitz kann die Grundversorgung aus dem Agrarbereich decken. Eine Infrastruktur mit asphaltierten Strassen, elektrischer Energie und Wasser ist ebenso vorhanden wie Schulen und Sanitätsstationen.

Geringe Erträge, kein möglicher Schuldenabbau und mangelndes Kapital bei fehlenden, zu beleihenden Grundbesitztiteln erforden illegale Nutzungen der Waldflächen und verhindern auf den Agrarflächen eine nachhaltige Agrartechnik. Die seit den Rodungen der sechziger Jahre angewandte traditionelle Landnutzung mit Feuereinsatz, aber auch fremdfinanzierter maschineller Zuckerrohr- und Maisanbau haben unter Missachtung der monsunal gesteuerten Abflussverhältnisse bei dem vorhandenen Relief, den anstehenden Gesteinen und den vorhandenen Böden in wenigen Jahrzehnten zu extremen Erosionsschäden mit teilweiser Entfernung der Böden oder Erosion mit Freilegung der Plinthithorizonte (Lateritkrusten) geführt - Prozesse, die nicht abgeschlossen sind und die stetig das Ertragspotential mindern. Dieses hat bereits zu Abwanderungen und Arbeitsmigration geführt.

Die Befunde zeigen in den Agrargebieten ein geschädigtes Ökosystem, das bei Fortführung der bisherigen Wirtschaftsweise weiter degradiert wird. Damit werden letztlich die geringen Einkünfte und auch die Versorgung selbst in Frage gestellt und die regionalen Disparitäten zu andern Räumen Thailands noch verstärkt.

\section{Perspektiven}

Die Befunde sind in allen wesentlichen Parametern, die für einen wirtschaftlichen Aufschwung, für die Beseitigung der Disparitäten und eine nachhaltige Bewirtschaftung entscheidend sind, nicht ermutigend. Die unkontrollierte Agrarkolonisation des Raumes mit der vorhandenen kapitalarmen Sozialstruktur hat ein labiles, sich rapide verschlechterndes Ökosytem und auch Wirtschaftssystem bewirkt.

Eine Stabilisierung oder gar ein Aufschwung kann nicht aus der Region selbst kommen.

Neue, an das monsunale Regime angepasste Agrartechniken, besonders für den großräumigen Zuckerrohr- und Maisanbau, sind nötig und auch in der Initialphase zu finanzieren.

Durch lokale Massnahmen - wie die 1995 erfolgte staatliche Unterstützung für die Errichtung einer Gummibaumpflanzung, die seit den 80er Jahren erfol- 
gende lokale Obstkulturförderung oder von der staatlichen Elektrizitätsbehörde gestiftete Obstbäume - wird versucht, einen Wandel in der Wahl der Anbauprodukte herbeizuführen. Dies sind erkennbare Einzelmaßnahmen, die wohl zukunftsweisend sind, aber noch keine Auswirkungen erkennen lassen.

Wesentlich wird auch eine bessere Ausnutzung der Wasserkapazitäten sein. Die «wohlhabenden» Orte liegen im Einzugsgebiet ganzjährig schüttender Quellen. Dort wachsen in den Hausgärten sonst in der Region nicht anbaubare Pflanzen aus immerfeucht tropischen Gebieten und die Bewässerung erbringt gute Erträge - und dies, obwohl es sich um Teile der Khoratrumpffläche mit ungünstigen Böden handelt. Begehungen in den «Oasen» zeigen, dass durch eine Mischbepflanzung eine ganzjährige Abschirmung der Landoberfläche erfolgt und die Monsunregen keine Erosionsgefährdung hervorrufen.

Die in den letzten Jahren zu beobachtenden Neuanlagen von Wasserbecken sowie ein kleiner neuer Staudamm am Rande von Thung Lui Lai dokumentieren staatliche Maßnahmen in diese Richtung. Mit Fremdkapital erfolgte Neuanpflanzungen von Baumkulturen deuten einen möglichen Wandel in der lokalen Agrarlandschaft an.

Insgesamt können aber nur größere, wohl staatliche Maßnahmen für einen Umschwung sorgen. Das große Interesse der thailändischen Behörden und die vielfältige Unterstützung der bisherigen Bestandsaufnahmeforschungen lassen hoffen, allerdings ist der Umfang der Maßnahmen und der Möglichkeiten des thailändischen Staates sicher ein limitierender Faktor.

\section{Literatur}

BLÜTHGEN, J. (1966): Allgemeine Klimageographie. 2. Auflage, Berlin: Walter de Gruyter: 118, Tab. 32.

Forstabteilung Chaiyaphom (1996): Amtlicher Bericht der Forstabteilung der Province Chaiyaphom an den Minister am 2. Juni 1996. - Chaiyaphom Province, Chaiyaphom: 12 (in Thai).

Khon San Agricultural Section (1995): Agricultural Development Plan. - Amphoe Khon San, Chaiyaphom Province, Chaiyaphom: 16 (in Thai).

Kunurat, P. (1987): Geography of Isaan. - Social Sciences Department, Khon Kaen University, Khon Kaen: 347 (in Thai).

LÖFfler, E. \& I. MAASS (1992): Das Khorat Plateau - Thailands Ungunstraum. - In: Geographische Rundschau 44: 57-64, Braunschweig.

Mo TA CHeK (1996): Gor Gor Cho 2 Kor Ban Mo Ta Chek. - Amphoe Khon San, Chaiyaphom Province, Chaiyaphom: 24 (in Thai).

Non Champa (1996): Gor Gor Cho 2 Kor Ban Non
Champa. - Amphoe Khon San, Chaiyaphom: 24 (in Thai).

Nong Chiang Rot TAI (1996): Gor Gor Cho 2 Kor Ban Nong Chiang Rot Tai. - Amphoe Khon San, Chaiyaphom: 24 (in Thai).

Pendleton, R.L. (1963): Thailand - aspects of landscape and life. - New York: Duell, Sloan and Pearce.

Regionale ForstabteILUng NaKHON RatchaSima (1973): Amtlicher Bericht der Regionalen Forstabteilung Jahr 1973. - Nakhon Ratchasima, Thailand (in Thai).

Regionale Forstabteilung NakHon Ratchasima (1974):Amtlicher Bericht der Regionalen Forstabteilung Jahr 1974. - Nakhon Ratchasima, Thailand (in Thai).

Regionale Forstabteilung NaKhon Ratchasima (1977): Amtlicher Bericht der Regionalen Forstabteilung vom 18. Oktober 1977. - Nakhon Ratchasima, Thailand: 6 (in Thai).

Regionale Forstabteilung Nakhon Ratchasima (1978): Amtlicher Bericht der Regionalen Forstabteilung vom 10. Februar 1978. - Nakhon Ratchasima, Thailand: 10 (in Thai).

Regionale Forstabteilung NaKhon Ratchasima (1979): Amtlicher Bericht der Regionalen Forstabteilung Jahr 1979. - Nakhon Ratchasima, Thailand (in Thai).

Regionale Forstabteilung Nakhon Ratchasima (1980): Amtlicher Bericht der Regionalen Forstabteilung vom 8. März 1980. - Nakhon Ratchasima, Thailand: 7 (in Thai).

Regionale Forstabteilung Nakhon Ratchasima (1983): Amtlicher Bericht der Regionalen Forstabteilung vom 8. Februar 1983. - Nakhon Ratchasima, Thailand: 6 (in Thai).

Regionale Forstabteilung Nakhon Ratchasima (1984): Amtlicher Bericht der Regionalen Forstabteilung vom 16. Oktober 1984. - Nakhon Ratchasima, Thailand: 6 (in Thai).

Regionale Forstabteilung Nakhon Ratchasima (1985): Amtlicher Bericht der Regionalen Forstabteilung vom 28. Juli 1985. - Nakhon Ratchasima, Thailand: 7 (in Thai).

Regionale Forstabteilung Nakhon Ratchasima (1989):Amtlicher Bericht der Regionalen Forstabteilung Jahr 1989. - Nakhon Ratchasima, Thailand (in Thai).

Royal Forestry Department (1984): Forest fire and control in Thailand. - Bangkok: Ministry of Agriculture and Cooperatives: 14 (in Thai).

Royal Forestry Department (1991): Sor Tor Gor Handbook. - Ministry of Agriculture and Cooperatives, Bangkok: 22 (in Thai).

Royal Forestry Department (1993): Thai Forestry Sector Master Plan Volume 2: Executive Summary and Proposed. - Forestry Sector Policy, Ministry of Agriculture and Cooperatives, Bangkok.

Royal Forestry Department (1994): Forest Statistics Year Book 1994. - Ministry of Agriculture and Cooperatives, Bangkok (in Thai).

Royal Forestry Department (1996): Forest Statistics 
Year Book 1996. - Ministry of Agriculture and Cooperatives, Bangkok (in Thai).

Royal Forestry Department (1998): Forest Statistics Year Book 1998. - Ministry of Agriculture and Cooperatives, Bangkok (in Thai).

SCHELL,A. (2001): Geoökologische Grundlagen der Landnutzung in einem Karstgebiet NE Thailands - Bodensequenzen,Standorteigenschaften und Reliefentwicklung am Beispiel des Amphoe Phu Pha Man. - Dissertation an der Geowissenschaftlichen Fakultät der Universität Tübingen. UhLIG, H. (1984): Spontaneous and planned agricultural settlements - a general view of the present clearing-colonization in the Asean countries of South East Asia. - In: Uhlig, H. (Hrsg.) (1984): Spontaneus and planned settlements in South East Asia: 6-118.

UhLIG, H. (1984): Spontaneous and planned settlements in South East Asia. - Hamburg: Institut für Asienforschung. Werner, W.L. (1993): Pinus in Thailand. - Geoecological Research 7.

YongVanit, S. (2001): Khon San - Petchaboon Mountains. Eine Fallstudie zu den Auswirkungen des wirtschaftenden Menschens auf ein tropisches Waldökosystem im Monsunklima Nordostthailands. - Dissertation an der Geowissenschaftlichen Fakultät der Universität Tübingen.

\section{Zusammenfassung: Khon San - eine Fallstudie zu Naturpotenzial und Agrarkolonisation in Nordost- thailand}

Im Nordosten Thailands, in der Region Isaan, fand in der zweiten Hälfte des letzten Jahrhunderts eine ungelenkte Agrarkolonisation statt. Im Rahmen eines deutsch-thailändischen Forschungsprojektes wurde Amphoe Khon San, am Westrand des Khoratplateaus, einer geoökologisch-sozioökonomischen Fallstudie unterzogen. Die Siedlungs- und Nutzungsgeschichte wurde rekonstruiert und im Vergleich mit geschützten noch wenig veränderten Waldgebieten wurden die Auswirkungen auf das Ökosystem bilanziert. Die Sozialstruktur der Orte und die wirtschaftliche Situation der Bewohner wurde aus Ortsdaten und Befragungen ermittelt. Die Möglichkeiten für zukünftige nachhaltige Entwicklungen wurden diskutiert.

\section{Summary: Khon San - a case study on environmental potential and agricultural colonization in Northeast Thailand}

In the Isaan, a region in the northeastern part of Thailand, uncontrolled agricultural settlement during the second half of the last century has been recorded. Within the framework of a research collaboration project between German and Thai scientists, an ecological and socio-economical case study was formulated. The goal of the study was to reconstruct the history of settlement and land use in Amphoe Khon San, situated on the western edge of the Khorat Plateau.The examined area was then compared with protected, almost untouched wooded areas in order to assess the impact of settlement and land use on the ecosystem. Local data of the villages and interviews with the inhabitants were used to obtain information about the social structure and the economic situation of the people. The possibilities open for future sustainable development were discussed.

\section{Résumé: Khon San - une étude de cas du potentiel naturel et de la colonisation agraire dans le Nord-Est de la Thailande}

Au nord-est de la Thaillande, dans la région Isaan, se déroulait pendant la deuxième moitié du siècle passé une colonisation agraire non-organisée. Dans le cadre d'un projet de recherche allemand-thaillandais, Amphoe Khon San, situé au bord occidental du plateau Khorat, fut soumis à une étude de cas écologique et socio-économique. L'histoire de la colonisation et de la mise en valeur fut reconstruite. En comparaison avec des régions forestières protégées et encore peu modifiées, un bilan fut dressé des répercussions sur le système écologique. $\mathrm{La}$ structure sociale des villages et la situation économique des habitants furent analysées à l'aide de données statistiques locales et d'enquêtes. Les possibilités de développements durables futurs furent évaluées.

\section{Didaktische Hinweise}

- Welches geoökologische Potenzial trafen die Siedler am Westrand des Khoratplateaus an?

- Was sind die Folgen einer nicht an die Naturbedingungen angepassten Landwirtschaft?

- Warum erfolgt Arbeitsmigration?

- Wie zeigt sich die sozioökonomische Struktur der neugegründeten Siedlungen?

- Wie unterscheiden sich die Einkünfte in den einzelnen Dörfern?

- Welche Auswirkungen zeitigen Eukalyptus- und Teakholz-Aufforstungen?

- Welche Massnahmen könnten zu einer Stabilisierung resp. einem Aufschwung der Region führen?

Prof. Dr. Karl-Heinz Pfeffer, Geographisches Institut, Universität Tübingen, Hölderlinstraße 12, D-72074 Tübingen.

e-mail:prof.pfeffer@t-online.de

Associate Prof. Dr. Sekson Yongvanit, Department of Social Sciences, Faculty of Humanities and Social Sciences, Khon Kaen University, Khon Kaen 40002, Thailand.

e-mail: sekyon@kku.ac.th

\section{Manuskripteingang/received/manuscrit entré le} 27.2.2002

Annahme zum Druck/accepted for publication/accepté pour l'impression: 16.2.2004 